\title{
Sobrevivência em Redes Ópticas: Protegendo a Proteção
}

\author{
Alisson Barbosa de Souza, Ana Luiza de B. de P. Barros, Antônio Sérgio de S. \\ Vieira, Jéssyca Alencar L. e Silva, Joaquim Celestino Júnior
}

\begin{abstract}
Resumo-A sobrevivência em Redes Ópticas WDM tem sido um dos assuntos de maior interesse nos últimos tempos. A idéia usada ao longo dos anos é estabelecer caminhos de proteção para que em eventos de falha esses caminhos possam ser utilizados. Entretanto, essas propostas não levam em conta a real situação em que os caminhos de proteção se encontram, de maneira que se possa atender as exigências das aplicações que os demandam. Neste artigo, propõe-se uma abordagem que leva em conta esta exigência, utilizando Algoritmo Genético e Lógica Fuzzy, considerando-se, ainda, as restrições de comprimento de onda e o SRLG (Shared Risk Link Group) dos enlaces.
\end{abstract}

Palavras-Chave-Redes ópticas, Algoritmos Genéticos, SRLG, proteção.

Abstract-The survivability in WDM optical networks has been one of the subjects of great interest recently. The idea used over the years, is to establish a protection path, in case of failure, these path can be used. However, these proposals do not take into account neither the real situation of these paths or the applications requirements. In this article, it is proposed an approach that takes into account this requirement, using genetic algorithm and fuzzy logic, considering addition, the restrictions of wavelength and SRLG (Shared Risk Link Group).

Keywords-Optical networks, Genetic Algorithms, SRLG, protection.

\section{INTRODUÇÃO}

As fibras ópticas têm se mostrado uma grande revolução como meio de transmissão. Suas baixas taxas de erro, sua capacidade de transmissão e sua imunidade aos ruídos são tidas como responsáveis por sua popularização. Cada vez mais redes de fibras ópticas se fazem presentes na ligação com outras redes e como redes de grandes corporações. Redes desse tipo devem funcionar 24 horas por dia, 7 dias por semana, para dar suporte a aplicações que exigem funcionamento ininterrupto. Tais como: aplicações de companhias aéreas, hospitais, bancos, supermercados e muitas outras.

A tecnologia WDM (Wavelenght Division Multiplexing), permite que redes ópticas possam suportar velocidades próximas de terabits por segundo e, conseqüentemente, qualquer interrupção pode levar à perda de enormes quantidades de dados, fazendo com que as aplicações sejam prejudicadas. Para que estes problemas sejam evitados, são necessárias técnicas de proteção seguras e confiáveis.

Uma das técnicas empregadas em redes ópticas para tentar garantir que seus serviços não sejam suspensos é o uso de

LARCES - Laboratório de Redes de Computadores e Segurança, Universidade Estadual do Ceará, Fortaleza, Brasil, E-mails: alisson@larces.uece.br, analuiza@larces.uece.br, sergiosvieira@larces.uece.br, jessyca@larces.uece.br, celestino@larces.uece.br. caminhos de proteção. Nesta técnica, utilizam-se caminhos alternativos (rotas de proteção) previamente definidos, para que, em caso de falha do caminho principal, seu fluxo não seja prejudicado, pois o tráfego é rapidamente redirecionado para sua rota de proteção.

Neste artigo, propõe-se um método para tentar assegurar que um caminho de proteção pré-estabelecido seja adequado em caso de falha do caminho principal. O tráfego deve ser desviado rapidamente e os enlaces da rota de proteção devem possuir baixa taxa de erro de bit $(B E R)$ e serem diferentes dos enlaces do caminho principal (link-disjunto).

Por utilizar redes ópticas WDM sem conversores de lambdas, neste artigo, uma outra questão a ser considerada é a continuidade do comprimento de onda, onde um lambda deve estar disponível fim-a-fim.

Para resolução destes problemas, algoritmos exatos, como Dijkstra, podem ser inadequados, pois só possuem resolução em tempo computacional razoável para pequenas instâncias [2], caracterizando-se como um problema NP-Completo. Em geral, para resolução desta classe de problemas são utilizadas meta-heurísticas que, apesar de não garantirem o ótimo, oferecem baixo tempo de resolução [3].

Utiliza-se neste trabalho Algoritmo Genético com apoio de Lógica Fuzzy, possibilitando que o método convirja rapidamente a uma solução ao mesmo tempo em que tenta superar as restrições impostas pelo problema.

O trabalho está organizado da seguinte forma: na Seção II são apresentados os trabalhos relacionados. A definição e formulação do problema estão respectivamente nas seções III e IV. A descrição do método é apresentada na Seção V. Em seguida, na Seção VI, são apresentados as análises e resultados. Por último, conclui-se o artigo na Seção VII.

\section{Trabalhos Relacionados}

Devido ao caráter NP-Completo apresentado por tais problemas, diversas maneiras de resolvê-los têm sido propostas. $\mathrm{Na}$ maioria dos trabalhos encontrados, não é considerado a manutenção do caminho de proteção.

No trabalho realizado em [4] foi desenvolvido uma heurística para alocar caminhos de proteção pré-planejados numa rede óptica WDM minimizando a capacidade excedente de recursos. O esquema proposto em nosso artigo, também leva em conta a minimização de uso dos recursos da rede, porém, utilizamos meta-heurística para alocar novos caminhos de proteção levando-se em conta classes de serviço.

Em [5] é analisado o cálculo de pares de caminhos disjuntos com a restrição do comprimento de onda. Este estudo 
mostra que as versões link-disjunto e nó-disjunto do problema são NP-Completo. O artigo faz uso de dois algoritmos, um aproximativo e outro exato, para encontrar os caminhos. Diferentemente, nosso esquema utiliza uma meta-heurítica, o AG, além de incluir restrições de continuidade de lambda, $B E R$, tipo de proteção e restrições do grupo de enlaces com risco compartilhado.

Em [6] é proposto um esquema de proteção considerando a restrição do SRLG, nele é desenvolvida uma heurística baseada em APF (Active-Path-First) numa rede óptica WDM com conversão de lambdas e é observada uma melhora no índice de probabilidade de bloqueio. Entretanto, não são analisados a qualidade das rotas e nem seus tipos de proteção. Em nosso trabalho, também é considerada a restrição do SRLG, porém não há conversão de lambdas.

\section{Definição do Problema}

O problema a ser tratado consiste em assegurar, para uma rede óptica WDM sem conversão de lambda, um caminho SRLG-disjunto e link-disjunto que atenda as exigências das classes de serviço em relação a baixas taxas de erro e os tipos de proteção.

Em uma rede óptica WDM, cada fibra possui um conjunto $\Lambda$ com $n$ comprimentos de onda, $\Lambda=\left\{\lambda_{1}, \lambda_{2}, \lambda_{3}, \ldots, \lambda_{n}\right\}$. Em uma rede sem conversores de lambda, uma rota origem-destino somente será estabelecida caso exista o mesmo $\lambda_{i}$ disponível fim-a-fim. Além disso, para tentar assegurar a sobrevivência de uma rede óptica WDM sem conversão de lambda, o caminho principal deve ser link-disjunto ao caminho de proteção.

O valor de SRLG identifica o grupo de risco de um enlace. Uma falha em algum elemento do grupo acarreta a interrupção do funcionamento dos outros elementos. Portanto, também é necessário que a rota de proteção seja link-disjunta aos grupos de risco dos enlaces da rota primária.

Considere um cenário no qual, através de uma rede óptica, existem várias rotas estabelecidas e uma delas possui um caminho de proteção exclusivo $(1: 1)$. Neste caso, apesar de se utilizar mais recursos da rede, o tráfego será rapidamente encaminhado para seu caminho de proteção caso ocorra alguma falha no caminho principal (quebra de uma fibra, queda na alimentação de energia do comutador, etc). Porém, não existe garantia de que o caminho de proteção seja adequado, naquele instante, para receber o tráfego encaminhado, já que neste momento esta rota pode apresentar problemas na taxa de erro de bit, por exemplo.

Consideramos uma rota de proteção adequada quando ela atende os requisitos das aplicações, sendo capaz de cumprir as exigências contratadas (SLA - Service Level Agreements). Por exemplo, um cliente que contratou um serviço para tráfego VoIP deve ter garantias de que mesmo em caso de falha da rota principal, a rota de proteção é capaz de atender as restrições de atraso e BER inerentes a uma aplicação de tráfego de voz.

A solução para este problema exige que uma nova rota de proteção deve ser disjunta à rota primária e disjunta aos grupos de risco do caminho principal.

Se considerarmos $E$ o conjunto de enlaces do caminho de proteção, $P$ o conjunto de enlaces do caminho principal e $S$ o conjunto de enlaces que fazem parte do mesmo grupo de risco dos enlaces da rota primária, então $E \cap(P \cup S)=\emptyset$. A Figura 1 mostra, como exemplo, uma rota secundária linkdisjunta e SRLG-disjunta à rota primária. Neste exemplo, $E=$ $\{a-b, b-c, c-d\}, P=\{a-e, e-d\}$ e $S=\{b-e, e-g\}$, onde os grupos de risco $R 1=\{e-d, e-g\}$ e $R 2=\{a-e, b-e\}$ possuem enlaces dos conjuntos $P$ e $S$.

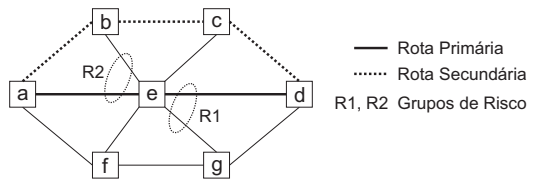

Fig. 1. Exemplo de Rota Primária e Secundária link-disjunta e SRLG-disjunta

Os valores de BER e tipos de proteção são utilizados para identificar um SLA contratado. Seguindo o proposto em [7], foram definidas quatro classes de serviço, e a cada uma delas, é atribuída uma faixa de BER. A Tabela I exibe os valores escolhidos para cada classe.

TABELA I

Classes de Serviço segundo Taxa de Erro de Bit

\begin{tabular}{|c|c|c|c|c|}
\hline Classe de Serviço & Gold & Silver & Bronze & Best-Effort \\
\hline Taxa de Erro de Bit & $\leq 10^{-8}$ & $\leq 10^{-7}$ & $\leq 10^{-6}$ & $>10^{-6}$ \\
\hline
\end{tabular}

Conforme [5], o problema de selecionar um caminho disjunto levando em conta a restrição de continuidade do lambda é NP-Completo. Neste artigo, além das mesmas restrições também é levado em conta a restrição do grupo de risco (SRLG). Pode-se concluir, portanto, que o problema aqui abordado também pode ser considerado NP-Completo.

\section{Formulação do Problema}

Uma rede WDM é modelada como um grafo conexo nãoorientado $G=(V, E, \Lambda, S)$, onde o conjunto de vértices $V=$ $\left\{v_{i} \mid i=1,2,3, \ldots, n\right\}$ representa os nós da rede, o conjunto de arestas $E=\left\{e_{i j} \mid v_{i}, v_{j} \in V\right\}$ representa os enlaces e $e_{i j}^{k}$ é a fibra $k$ que conecta os nós $v_{i}$ e $v_{j}$. Em cada enlace podem existir $m$ fibras, em cada fibra existe um conjunto de lambdas $\Lambda=\left\{\lambda_{1}, \lambda_{2}, \lambda_{3}, \ldots, \lambda_{n}\right\}$ e para cada lambda é definido um valor de taxa de erro de bit $-B E R \in[0,1]$, um tipo de proteção e se ele está disponível ou não.

$\mathrm{O}$ tipo de proteção define características relacionadas à utilização de um lambda. São definidos três tipos: (1) Never, (2) Shared e (3) Only. Um lambda com tipo de proteção Never não é utilizado em qualquer rota secundária, sendo possível utilizá-lo plenamente em um novo caminho de proteção $(1: 1$, $1+1$ ou $1: n$ ). Um lambda com tipo de proteção Shared é utilizado em uma rota secundária compartilhada e também pode ser utilizado na solução $(1: n)$. Já um lambda Only é utilizado exclusivamente por uma única rota primária e só deve ser escolhido caso não haja nenhuma outra solução possível.

Por último, $S_{i j}=\left\{s_{1}, s_{2}, s_{3}, . ., s_{n}\right\}$ define o conjunto de grupos de risco de $e_{i j}$. Onde $S_{i j}$ identifica os grupos de risco, aos quais $e_{i j}$ pertence.

Uma solução é considerada mais adequada, caso seja constituída por lambdas com baixo valor de $B E R$, tipo de proteção 
Never e seus enlaces devem ser link-disjunto e SRLG-disjunto à rota primária.

\section{Descrição da Proposta de Solução}

Para resolver o problema de escolha de uma nova rota de proteção optou-se por utilizar Algoritmos Genéticos (AG) e Lógica Fuzzy. O uso de meta-heurística, neste caso, é justificado por se tratar de um problema NP-Completo [5]. O uso de AG proporciona uma forma relativamente simples de representar várias métricas inerentes ao problema e comparar as soluções utilizando um valor de aptidão (fitness). Além disso, vários parâmetros de configuração do $\mathrm{AG}$ podem ser utilizados para achar uma solução que atenda as exigências do problema, por exemplo um tempo inferior a $50 \mathrm{~ms}$ [1]. O uso de Lógica Fuzzy é apropriado por representar melhor o valor de contribuição do $B E R$ no gene, conforme já demonstrado em [8].

A primeira medida tomada é a diminuição do espaço de busca da solução, ou seja, encontrar um subconjunto do conjunto de todas as rotas possíveis que atendam as restrições do problema.

Seja $U$ o conjunto de todos os enlaces da topologia, $P \subseteq U$ um subconjunto de enlaces da rota primária e $R \subseteq U$ um subconjunto de enlaces SRLG-não-disjunto a $P$. Antes de dar início à busca, são retirados todos os elementos de $U$ que fazem parte de $P$ e de $R$, assim como todos os lambdas dos elementos que possuem alto valor de BER. Desta forma, garante-se que a solução será link-disjunta e $S R L G$-disjunta ao caminho principal. Portanto, o conjunto $U^{\prime}=U-(P \cup U)$ representa o novo espaço de busca.

Um gene, nesta proposta, é uma tupla composta por uma aresta $\langle u, v\rangle \in U^{\prime}$ e por um $\lambda$ pertencente ao conjunto de lambdas da aresta $\langle u, v\rangle$. Portanto, um cromossomo é um conjunto $G=\left\{g_{1}, g_{2}, g_{3}, \ldots, g_{n}\right\}$ de genes. Um cromossomo será considerado inválido, ou seja, terá valor de fitness igual a zero, caso não possua o mesmo lambda em todos os seus genes (restrição para redes sem conversão de lambdas) ou se não formar uma rota válida origem-destino.

Neste artigo, para os casos válidos, o valor de fitness de um cromossomo $c$, com lambda $j$, será calculado segundo as formulações abaixo:

$$
\begin{aligned}
& \underline{\sum_{i=1}^{n} \alpha \cdot f\left(\text { ber }_{j}^{i}\right)+(1-\alpha) \cdot g\left(\operatorname{prot}_{j}^{i}\right)} \\
& \text { fitness }_{c}^{j}=\frac{f(0)+g(\text { Never })}{n^{2}} \\
& f(x)=\text { defuzzificador }(x) \\
& g(x)= \begin{cases}0,50, & \text { se } x=\text { Never } \\
0,49, & \text { se } x=\text { Shared } \\
0,01, & \text { se } x=\text { Only }\end{cases}
\end{aligned}
$$

Em (1), $n$ é a quantidade de genes do cromossomo $c$. O valor de $\alpha \in[0,1]$ define o peso de $B E R$ e tipo de proteção no problema. A expressão $f(0)+g($ Never $)$ representa o maior valor de contribuição de um gene (enlace ótimo).
Assim, o resultado de $\frac{\sum_{i=1}^{n} \alpha \cdot f\left(b e r_{j}^{i}\right)+(1-\alpha) \cdot g\left(\text { prot }_{j}^{i}\right)}{f(0)+g(\text { Never })}$ é o valor de contribuição relativo ao máximo valor de contribuição do gene. A divisão do somatório por $n^{2}$ é utilizada para dar maior valor de fitness aos menores cromossomos, minimizando o uso dos recursos da rede.

A função (2) retorna o valor de contribuição de $B E R$ do lambda $j$ do gene $i$ conforme [8].

A função (3) retorna um valor para o tipo de proteção do lambda $j$ do gene $i$. O valor de $g(x)$ influencia no resultado do problema ao dar maior valor de contribuição aos genes que possuem tipo de proteção Never ou Shared.

\section{A. Algoritmo Genético}

Nesta subseção é apresentado o funcionamento dos mecanismos que fazem parte do AG, onde cada um deles foi desenvolvido considerando as restrições impostas pelo problema. Os mecanismos desenvolvidos são: (I) População Inicial, (II) Seleção Natural, (III) Crossover e (IV) Mutação.

A população inicial (I) possui papel importante neste problema. Uma população inicial contendo boas soluções pode ajudar o AG a encontrar a melhor solução mais rapidamente do que utilizando uma populaçao inicial aleatória [3][9][10]. Portanto, o método de criação da população inicial deve gerar a maior quantidade de boas soluções no menor tempo possível. Neste método foram utilizadas duas abordagens. A primeira utiliza uma roleta viciada e a segunda uma roleta justa. Em ambas, $V$ é o conjunto de vértices adjacentes ao vértice $u$.

Na roleta viciada, o valor de $\Gamma_{v \in V}\left(u, v_{j}\right)$ (4) representa a porcentagem de área na roleta que a partir do vértice $u$ podese escolher o vértice $v_{j}$ adjacente. Ainda em (4), o valor de $n$ representa a quantidade de lambdas da fibra (aresta) $\left\langle u, v_{j}\right\rangle$ e $m$ a quantidade de arestas incidentes a $u$. A função $h(x)(5)$ retornará 1 caso $\lambda_{i}$ esteja disponível e 0 caso contrário. $\mathrm{O}$ valor retornado será multiplicado pelo peso dado ao tipo de proteção (3) do $\lambda_{i}$, assim, será concedido maior área na roleta aos enlaces que possuirem mais lambdas disponíveis e que tiverem tipo de proteção de maior peso. A Figura 2 exibe a idéia da montagem da roleta viciada.

$$
\begin{gathered}
\Gamma_{v \in V}\left(u, v_{i}\right)=\frac{\sum_{i=1}^{n} h\left(\lambda_{i}\right) \cdot g\left(\lambda_{i}\right)}{\sum_{j=1}^{m} \sum_{i=1}^{n} h\left(\lambda_{i j}\right) \cdot g\left(\lambda_{i j}\right)} \\
h(x)=\left\{\begin{array}{lll}
1, & \text { se } x & \text { está disponível } \\
0, & \text { se } x & \text { não está disponível }
\end{array}\right.
\end{gathered}
$$

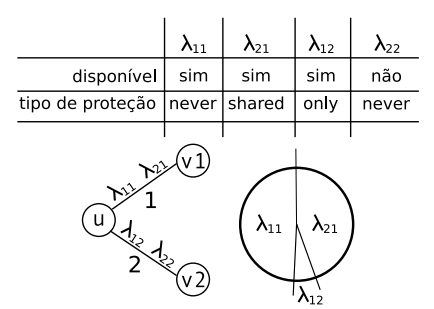

Fig. 2. Roleta Viciada 
Já o funcionamento da roleta justa se dá ao particionar a roleta em áreas iguais para cada lambda disponível entre os vértices $u$ e $v_{j}$. Portanto, caso existam duas fibras (enlaces) contendo dois lambdas disponíveis em cada uma, a roleta será dividida em quatro partes iguais. A Figura 3 exibe a idéia da montagem da roleta justa.

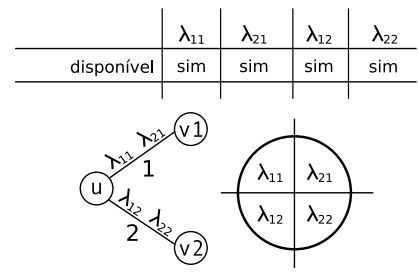

Fig. 3. Roleta Justa

Uma rota, na população inicial, é criada a partir do vértice de origem, escolhendo-se o vértice adjacente através de uma das roletas. Para cada novo vértice escolhido, realiza-se o procedimento anterior até que se encontre o destino da rota ou não existam mais vértices para serem escolhidos. Por último, atribui-se um lambda à rota criada utilizando um algoritmo de atribuição (FirstFit ou MostUsed).

Quando a população inicial for totalmente criada, ela será composta por rotas válidas e inválidas. Dentre as válidas, existirão soluções (cromossomos) com diferentes lambdas. Para melhor organizar os cromossomos na população inicial e para garantir que um cruzamento aconteça somente entre cromossomos com o mesmo lambda, foram criados grupos de lambdas. Para cada lambda existente na rede, um grupo é criado. Assim, se existirem 4 lambdas existirão 4 grupos e cada grupo será formado por soluções com lambda igual ao lambda do grupo.

O método de seleção natural utiliza os grupos de lambdas formados. A cada seleção são escolhidas a melhor solução e uma outra aleatória de um mesmo grupo. Para $n$ grupos e $m$ seleções, com $m>n$, a primeira seleção utiliza o grupo $\lambda_{i=1}$, a segunda o grupo $\lambda_{i=2}$ e assim sucessivamente até o grupo $\lambda_{i=n}$. Para as seleções onde $i>n$ serão utilizados novamente os grupos $\lambda_{i=1}$ até $\lambda_{i=n}$. Este método é responsável por escolher os melhores cromossomos que serão utilizados no crossover e mutação. Assim, garante-se que boas soluções não sejam perdidas a medida que novas gerações forem criadas.

A mutação é realizada no melhor cromossomo (rota) de um grupo. A partir do vértice central $b$, do cromossomo 1, tentase localizar um caminho alternativo até o vértice $c$, destino da rota, gerando com isto, uma nova solução, o cromossomo 2. Em seguida, no cromossomo 2, tenta-se atribuir um novo comprimento de onda. Caso a fitness do novo cromossomo seja maior do que a fitness do cromossomo original, este é inserido no grupo da próxima geração, caso contrário, é incluído o cromossomo original. A Figura 4 mostra um exemplo de mutação.

O crossover é realizado entre o melhor indivíduo e outro aleatório de um mesmo grupo retornados pela seleção natural. Primeiramente, tenta-se localizar vértices iguais no dois cromossomos para realizar o cruzamento. No caso da Figura 5,

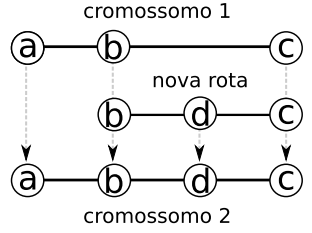

Fig. 4. Exemplo de Mutação

os cromossomos 1 e 2 possuem o vértice $b$ em seus genes. $\mathrm{O}$ filho 1 será constituído pelos vértices da origem até o vértice $b$ do cromossomo 1 e pelos vértices subseqüentes de $b$ até o destino do cromossomo 2. De forma semelhante, o filho 2 será constituído pelos vértices do cromossomo 2 da origem até $b$ e pelos vértices do cromossomo 1 após $b$ até o destino. Caso não existam vértices iguais nos cromossomos o melhor indivíduo é passado para o grupo da próxima geração. Observe que por se tratarem de cromossomos do mesmo grupo não é necessária uma nova atribuição de $\lambda$.

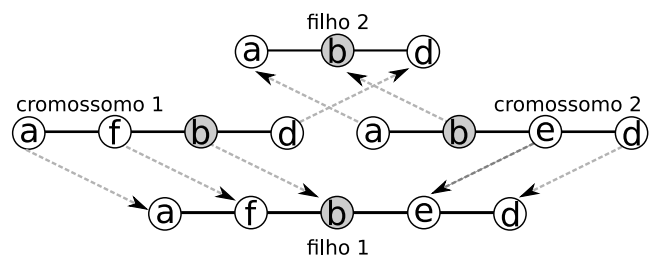

Fig. 5. Exemplo de Crossover

\section{Análises e Resultados}

Os experimentos foram realizados utilizando um cenário hipotético de uma rede para a América do Sul (Figura 6), que é composta por 52 nós e 99 enlaces. Cada enlace possui apenas uma fibra e cada fibra quatro lambdas. Cada um dos lambdas possui um tipo de proteção especificado (Only, Shared, Never), um respectivo valor de $B E R$ e se ele está disponível ou não.

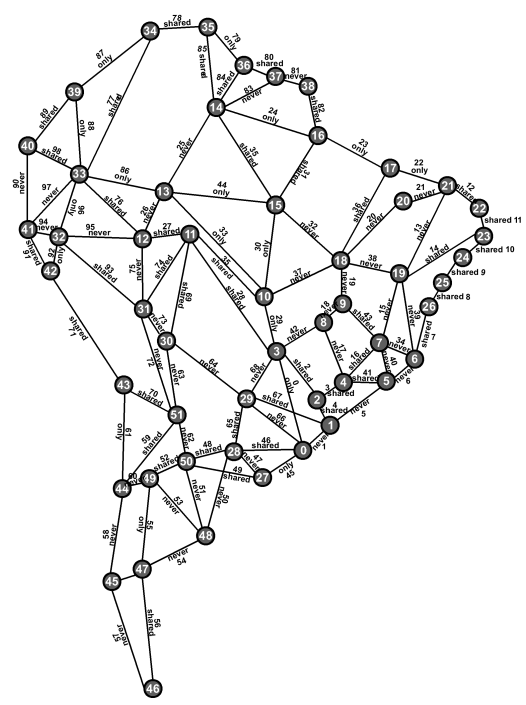

Fig. 6. Topologia Hipotética da América do Sul utilizada dos testes

Neste cenário, cerca de $70 \%$ dos lambdas tem valores de BER adequados (aproximadamente $10^{-9}$ ) para fornecer 
qualidade de serviço para as classes Gold, Silver e Bronze e o restante possui alto valor de $B E R\left(>10^{-3}\right)$. Além disso, alguns enlaces são SRLG conjunto aos enlaces do caminho principal para dificultar a escolha de soluções sub-ótimas.

Nos testes realizados neste artigo foram considerados quatro fatores: o tempo de busca da solução, a qualidade (valor da fitness) da solução, o tipo de roleta utilizada na criação dos indivíduos e a probabilidade de bloqueio.

Além do mais, é feita uma comparação entre a qualidade da rota selecionada por este algoritmo e a qualidade da rota calculada pelo algoritmo de Dijkstra.

De início simulou-se uma quebra de pré-requisito, alto valor de $B E R$, no caminho de proteção de uma determinada rota. Para tanto, em determinado momento da simulação atribuise explicitamente um valor de $B E R>10^{-3}$ para um lambda de uma rota de proteção de um tráfego Silver (requer $B E R \leq$ $10^{-7}$ ). Assim, o algoritmo buscará uma nova rota de proteção.

Para os experimentos de tempo do cálculo da nova rota de proteção, foi usada uma máquina Core 2 Duo, $2.2 \mathrm{GHz}$ e $2 \mathrm{~GB}$ de memória RAM. Foram analisados alguns parâmetros que influenciam no tempo de resposta do algoritmo: tamanho da população e tipo de roleta. Na Figura 7, percebe-se que em uma população com 512 indivíduos a média de tempo fica em torno de $50 \mathrm{~ms}$ e para uma população com 256 indivíduos, essa média fica em torno de $25 \mathrm{~ms}$. Para garantir que o tempo total do algoritmo não ultrapasse os $50 \mathrm{~ms}$ optou-se por uma população com 256 indivíduos.

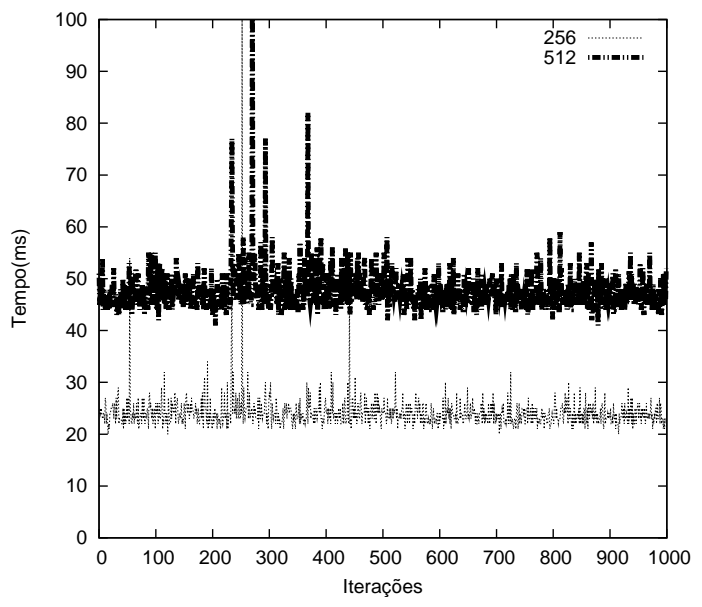

Fig. 7. Tempo para diferentes tamanhos de população

Nos experimentos foram utilizados dois tipos de roleta, justa e viciada, conforme detalhado na Seção V-A. Usando a roleta viciada para criar os cromossomos da população inicial, obteve-se ganho na qualidade das soluções encontradas, conforme descrito a seguir.

Para dizer que uma solução é a melhor, ou pior, de todas, existe a necessidade de comparar esta solução com todas as outras possíveis soluções. Isto não é feito em tempo polinomial; existe um número exponencial de possíveis combinações de comparações de rotas - semelhante ao problema do caixeiro viajante [11]. Para avaliar a qualidade das rotas em tempo polinomial, utilizou-se um novo critério que faz uso de uma amostra das possíveis soluções. O algoritmo é executado diversas vezes e a cada execução a solução é armazenada em um vetor. Ao final, o vetor é ordenado pelo valor de fitness. Dessa forma, as soluções com os menores índices do vetor são as que têm menores valores de fitness. Como as definições "melhor", "média" e "pior" são um tanto imprecisas quando não se pode comparar todas as combinações de soluções, decidiu-se por dividir o vetor em três partes iguais. Uma solução é dita ser "pior" se estiver na parte inicial do vetor, "média" na segunda parte e "melhor" na parte final do vetor.

De fato, analisando os resultados obtidos, os indivíduos que ficaram na porção final do vetor, com os melhores valores de fitness, têm os mais baixos valores de BER, bons tipos de proteção e são rotas curtas.

Conforme as Figura 8 e 9, foram feitos 1000 experimentos para cada configuração de parâmetros e contadas quantas soluções foram melhores, médias e piores. Constatou-se que, na configuração usada pelo $\mathrm{AG}$, o algoritmo mostra ser eficiente e retorna, em grande parte das vezes, as melhores soluções. Além disso, usando roleta viciada há um maior índice de escolha de melhores rotas.

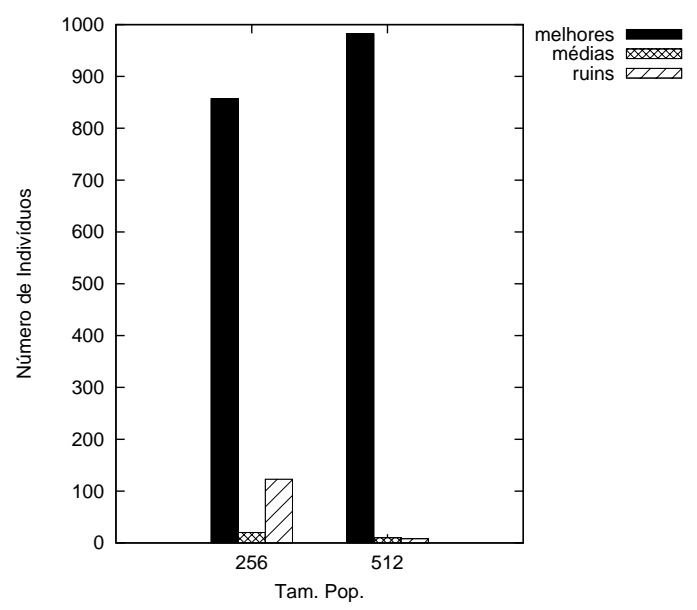

Fig. 8. Qualidade das soluções retornadas (Roleta Viciada)

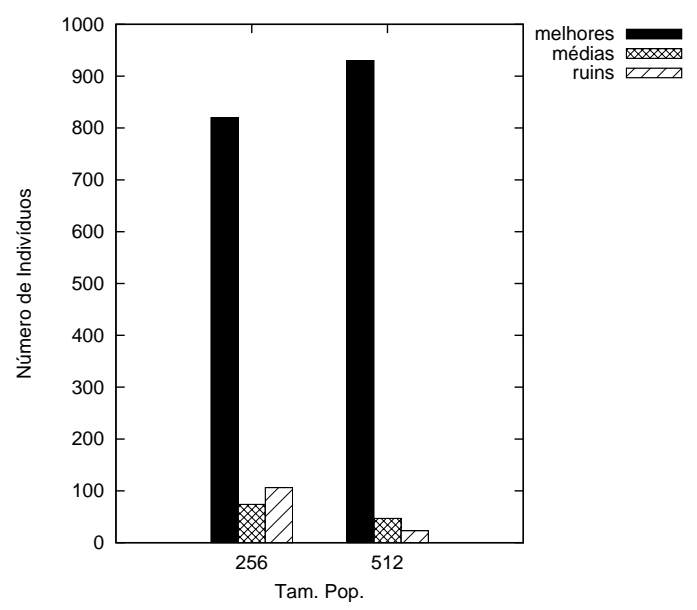

Fig. 9. Qualidade das soluções retornadas (Roleta Justa)

Para validar a eficácia do algoritmo realizou-se uma comparação entre a solução retornada pelo algoritmo desen- 
volvido neste trabalho (DisjointSchema) e a solução obtida pelo algoritmo de Dijkstra. As Figuras 10 e 11 exibem alguns dos resultados.

No gráfico da Figura 10, o eixo $x$ representa a quantidade de iterações realizadas e o eixo $y$ o valor da fitness da solução encontrada.

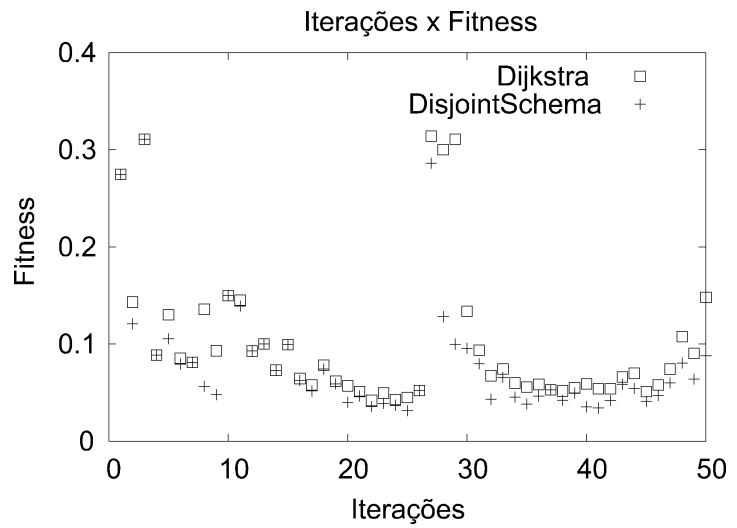

Fig. 10. Dijkstra x DisjointSchema - Iteração 0 a 50

Os resultados do gráfico da Figura 11 foram obtidos escolhendo-se aleatoriamente um destino para uma determinada origem fixa e a quantidade de lambdas de cada enlace foi variada de 10 a 100 . Como pode ser observado, o algoritmo de Dijkstra retorna a rota em tempo superior ao do DisjointSchema independente da quantidade de lambdas dos enlaces. Além disso, o DisjointSchema é capaz de encontrar a rota em tempo inferior a $50 \mathrm{~ms}$ para todas as quantidades de lambdas, enquanto o Dijkstra já ultrapassa esse tempo em testes com 40 lambdas.

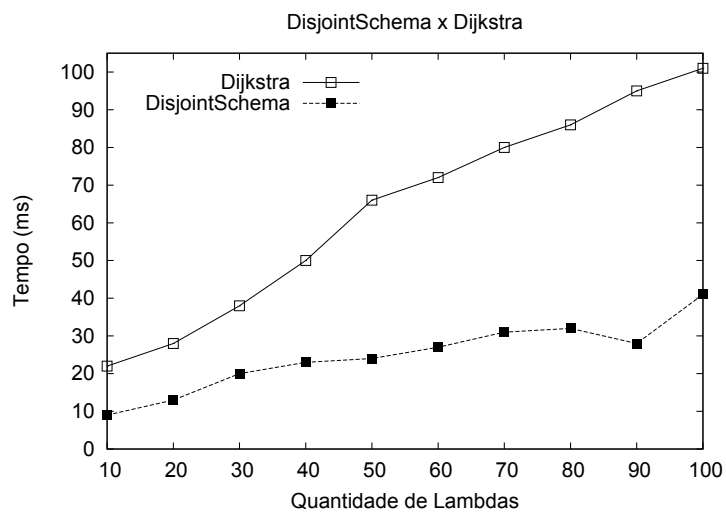

Fig. 11. Dijkstra x DisjointSchema - Tempo

Os gráficos exibem os valores ótimos obtidos pelo algoritmo de Dijkstra em comparação com as soluções conseguidas pelo DisjointSchema. Em média, o algoritmo proposto neste artigo, retorna soluções $81,40 \%$ do ótimo, porém com a vantagem de retornar o resultado em tempo computacional inferior ao tempo consumido pelo algoritmo de Dijkstra.

\section{CONCLUSÃO}

$\mathrm{O}$ artigo descreve um esquema de escolha de melhor rota de proteção link-Disjunta e SRLG-Disjunto em redes ópticas com restrição da continuidade do lambda, através do uso de Algoritmos Genéticos com apoio da Lógica Fuzzy.

$\mathrm{O}$ esquema proposto alcança resultados bastante satisfatórios contribuindo para a sobrevivência da rede ao mesmo tempo que procura satisfazer as exigências das aplicações. As rotas de proteção encontradas pelo método possuem enlaces com baixos valores de BER e tipo de proteção Never. Além disso, elas não compartilham do mesmo grupo de risco e dos enlaces do caminho principal. Também foi demonstrado através de comparações que os resultados se aproximam bastante da solução ótima encontrada pelo algoritmo de Dijkstra, com a vantagem de retornar o resultado em tempo computacional inferior.

Outra contribuição importante deste trabalho está na forma que o roteamento é realizado com AG. O sucesso do método está diretamente relacionado ao método de criação da população inicial por conseguir criar rapidamente boas soluções para o problema. Além disso, o esquema de grupos usado na população inicial permite comparar a fitness de qualquer tipo de rota, mesmo as rotas que têm os mesmos enlaces, mas que usam lambdas diferentes, para escolher a melhor solução.

\section{TRABALhos Futuros}

Os trabalhos futuros incluem o uso de outras metaheurísticas para a solução do problema. Além disso, serão utilizados outros parâmetros característicos das redes ópticas. Por fim, novas tecnologias de transmissão ópticas, como OBS (Optical Burst Switching), serão utilizadas.

\section{REFERÊNCIAS}

[1] J.P. Vasseur, M. Pickavet, P. Demeester, Network Recovery: Protection and Restoration of Optical, SONET-SDH, IP, and MPLS, Morgan Kaufmann Publishers Inc. San Francisco, CA, USA, 2004.

[2] R.Sedgewick, Algorithms, Addison-Wesley, 1988.

[3] F. Glover and G. Kochenberger, Handbook of Metaheuristics, volume 57 of International Series in Operations Research \& Management Science, Kluwer Academic Publishers, 2003.

[4] G. Maier, A. Pattavina, S. De Patre, M. and Martinelli, Optical Network Survivability: Protection Techniques in the WDM Layer, Photonic Network Communications, v. 4, number 3, pp. 251-269, Springer, 2002.

[5] R. Andersen, F. Chung, A. Sen, and G. Xue, On disjoint path pairs with wavelength continuity constraint in WDM networks, INFOCOM 2004. Twenty-third AnnualJoint Conference of the IEEE Computer and Communications Societies, v. 1, 2004.

[6] Z.L. Tang and X.M. Li, A Mixed Shared and Multi Paths Protection Scheme with SRLG Constraints, Proceedings of the Eighth ACIS International Conference on Software Engineering, Artificial Intelligence, Networking, and Parallel/Distributed Computing (SNPD 2007)-Volume 01, pp. 60-65, 2007,IEEE Computer Society Washington, DC, USA.

[7] C. Pinart, Alternatives for in-service BER estimation in all-optical networks: towards minimum intrusion,JOURNAL OF COMPUTERS, v. 2, number 3, pp. 55, 2007.

[8] J. Celestino Jr., A.B. Souza, A.S.S Vieira, J.A.L. Silva, A.L.B.P. Barros, G.A.L. Campos, and L.W.N. Mendouga, GAFUPA: Uma Proposta para Proteção em Redes Ópticas,XXVI Simpósio Brasileiro de Telecomunicações - SBrT, Rio de Janeiro, RJ, 2008.

[9] C.R. Reeves, A genetic algorithm for flowshop sequencing, Computers and Operations Research, v. 22, pp. 5-13, Elsevier, 1995.

[10] R.K. Ahuja, and J.B. Orlin, Developing Fitter Genetic Algorithms, INFORMS Journal on Computing, v. 9, pages 251-253, 1997.

[11] T.H. Cormen, C.E. Leiserson, R.L. Rivest, and C. Stein, Introduction to Algorithms, MIT Press, 2001.

[12] A. Wason and R.S. Kaler, Wavelength Assignment Problem in Optical WDM Networks, IJCSNS, v.7, pages 27, 2007. 